\title{
Altering Airflows and Stopping Drops
}

\author{
Two new studies provide insights into the efficacy of masks under \\ different usage conditions, results that could help improve strategies for \\ lowering transmission of COVID-19.
}

\section{By Katherine Wright}

S ince early in the COVID-19 pandemic, scientists have advised that masks could help control the virus, which can spread via drops of saliva emitted during breathing and speaking (see How Talking Spreads Viruses). But while masks have been qualitatively shown to be effective at reducing the spatial extent of drop-laden air flows, their impact on some airflows remains unquantified, and there are open questions about how effective different mask types are at halting drops. Now, Philippe Bourrianne of Princeton University and colleagues [1] and Abhishek Saha of the University of California, San Diego, and colleagues [2] present results that clarify these issues. The work could help improve strategies for lowering the risk of virus transmission through the air.

In the first study, Bourrianne and his colleagues considered how a mask changes the airflow pattern around a person as they exhale. The team performed infrared imaging experiments that revealed the carbon dioxide $\left(\mathrm{CO}_{2}\right)$ emitted when a maskless person breathed out. They then repeated the experiments for a person wearing a standard triple-ply surgical mask.
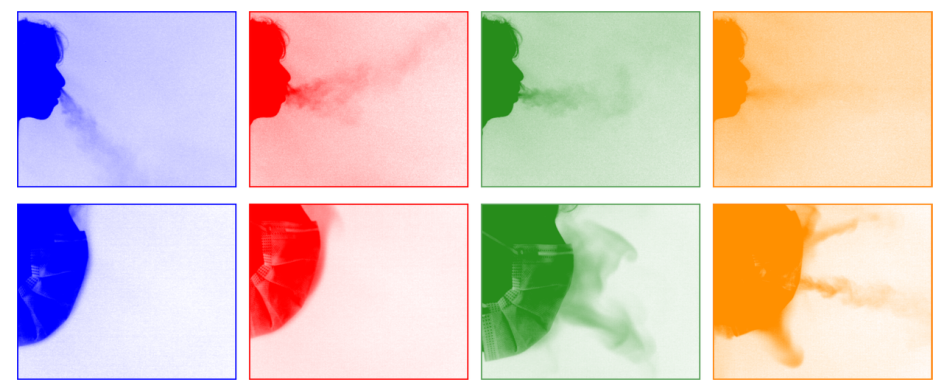

Credit: P. Bourrianne et al. [1]

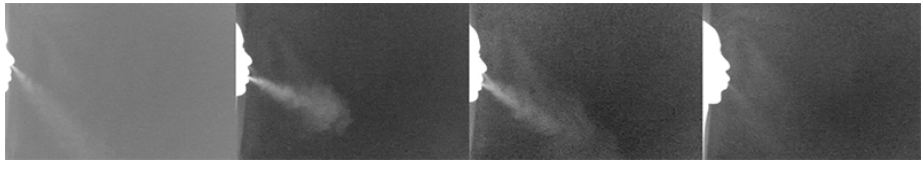

Video 1: Infrared video of a person breathing without a mask. (Left to right) Four kinds of breathing are pictured: soft with a closed mouth, soft with an open mouth, heavy breathing, and blowing. Credit: P. Bourrianne et al. [1]

In the absence of a mask, the team observed a fast-moving jet of $\mathrm{CO}_{2}$ that could extend for more than a meter. This jet was directed down toward the floor when the $\mathrm{CO}_{2}$ came from the person's nose and horizontally when the $\mathrm{CO}_{2}$ came from their mouth. The presence of the mask significantly altered these flow patterns. For gentle breathing, instead of a jet, the team observed a quasivertical cloud of $\mathrm{CO}_{2}$ that emanated from the top of the mask and traveled up the person's face. For heavy breathing, this quasivertical flow was accompanied by a few small jets that penetrated the mask. The extent of these jets, however, was much shorter than those produced by unmasked people, traveling a distance of around $10 \mathrm{~cm}$.

Bourrianne and his colleagues say that their results quantify how masks mitigate the direct transport of drops, which was previously unquantified. They note that the results suggest that safer indoor social interactions might be achieved by placing air-filtration systems above masked people that can remove virus-laden drops from the quasivertical flows the people produce. 

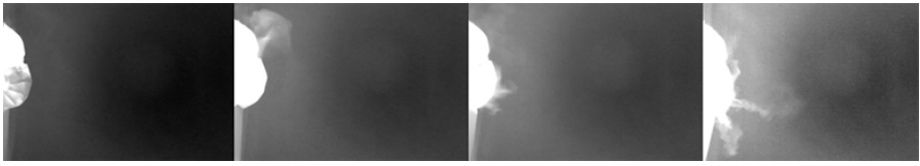

Video 2: Infrared video of a person breathing while wearing a mask. (Left to right) Four kinds of breathing are pictured: soft with a closed mouth, soft with an open mouth, heavy breathing, blowing. Credit: P. Bourrianne et al. [1]
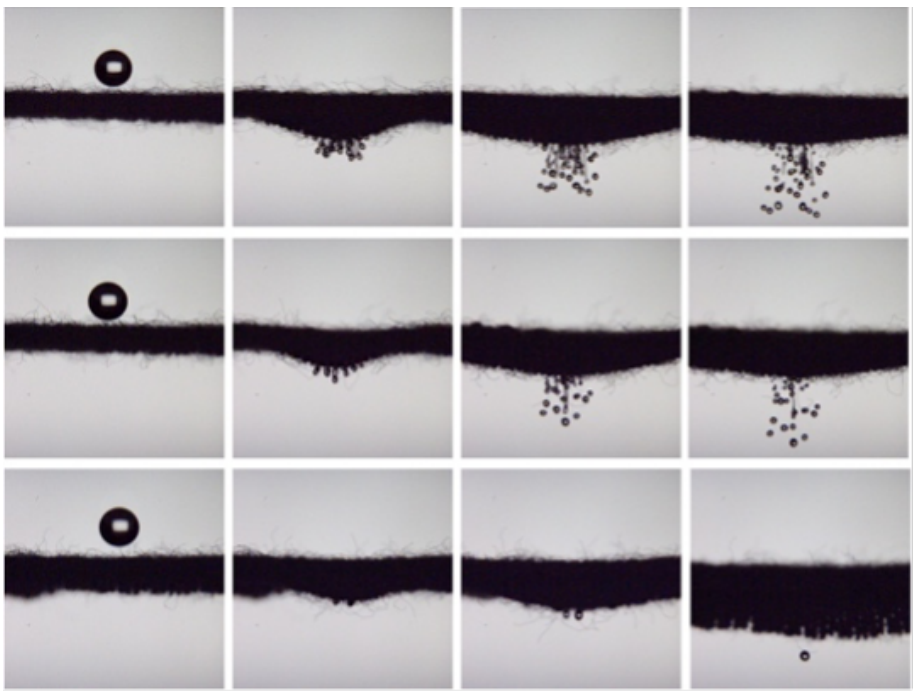

Figure 2: Photographs showing the penetration of drops through single-ply (top and middle) and triple-ply (bottom) masks. The top row of images shows a dry mask while the bottom two rows show wet masks.

Credit: A. Saha et al. [2]
In the second study, Saha and colleagues investigated how drops penetrate dry and wet masks. The team let a series of water drops fall onto single-ply masks. They used a high-speed camera to image the breakup of the drops into smaller drops (atomization) and measured the volume of water that made it through the mask. The work follows on from another recent study by the team, where they also looked at drops impacting three-ply masks.

The team found that single-ply masks were more likely than three-ply masks to atomize a drop. Their results also showed that, for both sets of masks, the masks became significantly better at blocking drops as they became wetter.

Saha notes that masks absorb fluid that condenses from the wearer's breath and sweat and, in humid environments, from the air; thus, understanding how a mask's efficacy changes as it gets wetter is vital. The results imply that wet masks do not necessarily need to be switched out with dry ones; in fact, doing that could reduce the mask's ability to protect. However, Saha notes that wetness may deteriorate other properties such as the mask's breathability and how well it fits, which the team did not measure.

Katherine Wright is the Deputy Editor of Physics.

\section{REFERENCES}

1. P. Bourrianne et al., "Quantifying the effect of a mask on expiratory flows," Phys. Rev. Fluids 6, 110511 (2021).

2. S. Bagchi et al., "Penetration and secondary atomization of droplets impacted on wet facemasks," Phys. Rev. Fluids 6, 110510 (2021). 